Abstract PS2-19

Enrollees in Deductible-Based Health Insurance Plans: Who Takes the Road Less Traveled?

Jie Huang, PhD, Kaiser Permanente; Mary Reed, DrPh, Kaiser Permanente; Richard Brand, PhD, University of California, San Francisco; John Hsu, MD, Kaiser Permanente

Background: Many employers are offering their employees choices of health insurance plans that differ in their upfront costs, e.g., lower monthly employee premiums, and in the amount of patient cost-sharing, e.g., deductibles for the first dollar of care. In a group of employees who had a choice of traditional plan (no deductible) or deductible plan in 2006, we examined characteristics associated with choosing the deductible plan. Methods: All subjects were primary subscribers in a large integrated health delivery system with a choice of traditional and deductible health plans. We used logistic regression to examine the characteristics associated with the choice of deductible plan and explored interactions between characteristics. Subscriber characteristics include enrolling any children (age $<18$ years) or elderly (65 years or older) on their plan, enrolling any chronic disease patients with asthma, diabetes, coronary artery disease, hypertension, or heart failure on their plan, neighborhood (census block group) education and income. There were 5 levels for neighborhood education: $=20 \%,=15 \%$, $=10 \%,=5 \%$ or $<5 \%$ of adults had less than a high school education, and 6 levels for neighborhood income: median household income $<150 \%,<200 \%$, $<300 \%,<400 \%,<500 \%$ or $=500 \%$ of the federal poverty level. Results: Among the 29,701 primary plan subscribers, $21 \%$ chose a deductible plan, $30 \%$ of the subscribers had children or elderly on their plan, and $38 \%$ had a chronic disease patient on their plan. Characteristics associated with choosing a deductible plan included not enrolling children or elderly on their plan $(\mathrm{OR}=1.32,95 \% \mathrm{CI}: 1.23-1.41)$, having no chronic patient on their plan $(\mathrm{OR}=1.61$, 95\% CI: 1.33-1.96), living in neighborhoods with higher education $(\mathrm{OR}=1.16$ for each level increase in education, $95 \% \mathrm{CI}$ : 1.13-1.18), living in neighborhoods with higher median income $(\mathrm{OR}=1.19$ for each level increase in income among subscribers having no chronic patient on the plan, 95\% CI: $1.15-1.23$; OR=1.13 for each level increase in income among subscribers having chronic patients on the plan, 95\% CI: 1.08-1.19). Conclusions: One in five employees with the choice of a deductible health insurance plan chose the deductible-based plan. Employees without children or elderly on their plan, who lived in neighborhoods with higher educational attainment and higher median household incomes, and who had no one with a chronic condition on their plan were more likely to choose a deductible plan.

\section{Abstract PS2-20 \\ Associations Between Self-Reported and Database Out-of-Pocket Drug Costs and Changes in Prescription Drug Behavior}

Claire E. Margerison, MPH, Kaiser Permanente Division of Research; Vicki Fung, PhD, Kaiser Permanente Division of Research; Mary Price, MA, Kaiser Permanente Division of Research; Richard Brand, $\mathrm{PhD}$, University of California at San Francisco; John Hsu, MD, MBA, MSCE, Kaiser Permanente Division of Research

Aims: We examined 2006 total self-reported and database-calculated out-of-pocket (OOP) costs and prescription drug behavior under Medicare Part $\mathrm{D}$ to assess the association between cost measures and behavior change. Methods: We collected information on 2006 total self-reported OOP costs and cost responses through a stratified random sample survey of Part D members of a large integrated delivery system (IDS) $(n=1040$, response rate $=74.9 \%$ ). Behavior changes included reduced adherence (e.g. skipping pills), cost-coping (e.g. switching to cheaper drugs), and financial burden (e.g. borrowing money). We also calculated total 2006 OOP costs using the IDS' pharmacy database. We used logistic regression to examine the association of self-reported and database OOP costs with behavior change, adjusting for gender, education, marital status, race/ethnicity, self-reported health, income, assistance with medications, and number of prescriptions in 2006. All analyses were weighted for sampling proportions. Results: Sixty-seven percent of patients reported costs $=\$ 500,16 \%$ reported $\$ 500-\$ 1000,10 \%$ reported $\$ 1000-\$ 2000$, and $8 \%$ reported $=\$ 2000$. Fifty-one percent of respondents reported OOP costs that agreed with their database OOP cost category, $18 \%$ reported lower costs, $32 \%$ reported higher costs. Patients taking 5 different medications and those with higher database OOP costs were less likely to report OOP costs in the same category as the database calculation. Overall, $36 \%$ of respondents reported 1 behavior change due to drug costs. Patients with self-reported OOP costs $>\$ 500$ had higher odds of reporting behavior change compared to those with costs $<\$ 500 \quad(\mathrm{OR}=3.4 \quad[1.8-6.2]$ for $\$ 500-\$ 1000, \mathrm{OR}=3.6 \quad[1.8-7.5]$ for $\$ 1000-\$ 2000, \mathrm{OR}=3.3$ [1.7-6.5] for $>\$ 2000$ ) after adjustment for database costs and covariates. Database OOP costs were not significantly associated with behavior change after adjustment for self-reported costs. Patients with income below $200 \%$ of federal poverty level and those with 5 prescriptions had higher odds of behavior change. Conclusions: Less than half of patients reported OOP costs in the same category as our database calculation; over 1-in-4 reported higher costs. Higher self-reported costs, but not database costs, were associated with behavior changes due to costs, and patients with lower incomes or more prescriptions may also be more likely to reduce adherence, switch to cheaper drugs, or experience financial burden due to costs.

\section{Abstract PS2-21 \\ Drug Use Among Medicare Subjects With a $\$ 1000$ Prescription Drug Benefit Cap}

Mary M. Price, MA, Kaiser Permanente Division of Research; Jie Huang, $\mathrm{PhD}$, Kaiser Permanente Division of Research; Richard Brand, PhD, University of California San Francisco; John Hsu, MD, MBA, MSCE, Kaiser Permanente Division of Research

Background/Aims: Prior studies suggest that patients with a drug benefit cap decrease their treatment adherence and have poorer outcomes compared to patients with no limit. We examined differences in drug choice, adherence, and burden between patients who exceeded their cap and those that did not, focusing on subjects using a drug from at least one of five widely used therapeutic drug classes. Methods: All subjects were continuously enrolled members of Kaiser Permanente Northern California (KPNC) in 2003, had a $\$ 1,000$ annual drug benefit cap, Medicare insurance, and were 65 years and older. We identified the five therapeutic drug classes most frequently used by these subjects in 2003 (anti-lipemics, beta adrenergic blockers, angiotensin converting enzyme inhibitors, narcotic analgesic combinations, and anti-ulcer drugs) and limited our analyses to patients with at least one dispensing from one of these classes in 2002. We assessed the mean number of study classes used and other prescriptions dispensed. We used logistic regression models to examine the association between exceeding the benefit cap and adherence and generic use within each class, adjusting for patient characteristics and drug burden. We also examined cost differences between drugs dispensed and the least expensive drug within the class. Results: Of the 97,922 subjects taking at least one of the study drugs, 15,926 (16\%) exceeded the $\$ 1000$ benefit cap in 2003 . We found that subjects who exceed the benefit cap had a greater overall drug burden than patients who did not exceed the cap (2.4 vs. 1.8 study classes used; 25.1 vs. 11.8 dispensing of other drugs). After adjustment, patients who exceeded the cap were more likely to be adherent to their drug treatment regimen, but less likely to be a generic user compared to patients who did not exceed the cap, (e.g., anti-lipemics: $74 \%$ vs. $69 \%$ adherent, $\mathrm{OR}=1.3,95 \% \mathrm{CI}: 1.2-1.4 ; 56 \%$ vs. $84 \%$ generic users, $\mathrm{OR}=0.30,95 \%$ CI: $0.28-0.31$ ). In all study classes, patients who exceeded the cap predominantly received a drug that was not the least expensive option within the class (e.g., $83 \%$ received a higher cost anti-lipemic, mean annual difference overall $=\$ 286$ ). Conclusions: Drug choice, adherence, and burden all are strongly associated with overall drug expenditures as expected. Many patients who exceed their available drug benefits, however, are not on the least expensive drug regimens available to them. 